\title{
Genomic analysis reveals RhoC as a potential marker in hepatocellular carcinoma with poor prognosis
}

\author{
W Wang', L-Y Yang*,', G-W Huang', W-Q Lu', Z-L Yang', J-Q Yang' and H-L Liu' \\ 'Liver Cancer Laboratory, Department of Surgery, Xiangya Hospital, Central South University, 87 Xiangya road, Changsha, Hunan Province 4I0008, \\ China
}

Hepatocellular carcinoma ( $\mathrm{HCC}$ ) is one of the most malignant human tumours because of its high incidence of metastasis. The mechanisms underlying the metastasis of HCC, however, remain poorly understood. In this study, we performed cDNA microarray analysis to profile gene expression patterns in two subtypes of HCC, solitary large HCC (SLHCC) and nodular HCC (NHCC), which differ significantly in the incidence of metastasis. Among 668 genes that were differentially expressed, we focused on RhoC, whose expression was significantly decreased in SLHCC compared to NHCC. The expression of RhoC in HCC and pericarcinomatous liver tissues (PCLT) was analysed at both the mRNA and protein levels by reverse transcription-polymerase chain reaction (RT-PCR) and Western blotting. In addition, immunohistochemistry was also performed on 94 cases of HCC with follow-up information. Collectively, our data indicate that the expression of RhoC significantly increased in HCC compared to PCLT; extrahepatic metastatic lesions expressed significantly higher levels of RhoC than the corresponding intrahepatic HCC tissues. There is a highly significant correlation of the RhoC expression levels with tumour vein invasion, number of tumour nodes and the status of differentiation. Significantly, the HCC patients with RhoC-positive expression had shorter survival than those with RhoC-negative expression. Together, our findings suggest a strong correlation between the expression of RhoC and HCC metastasis, implicating RhoC as a potential prognosis marker and therapeutic target for HCC.

British Journal of Cancer (2004) 90, 2349-2355. doi:I0.1038/sj.bjc.660I749 www.bjcancer.com

Published online II May 2004

(c) 2004 Cancer Research UK

Keywords: hepatocellular carcinoma; invasion and metastasis; prognostic marker; RhoC; cDNA microarray

Hepatocellular carcinoma (HCC) is one of the most common malignancies in Asia and Africa (Murray and Lopez, 1997; Akriviadis et al, 1998), especially in China. It is responsible for approximately one million deaths each year, predominantly in the developing countries (Schafer and Sorrell, 1999). During the past decade, hepatic resection for HCC has evolved into a safe procedure with low operative mortality (Makuuchi et al, 1998; Fan et al, 1999). However, the long-term survival remains unsatisfactory because of a high incidence of recurrence and metastasis after hepatic resection, with a 5-year actuarial recurrence rate of $75-100 \%$ reported in the literature (Poon et al, 2000). Most recurrence was attributed to invasion and metastasis of HCC, so the elucidation of prognostic factors reflecting invasion and metastasis not only provides guidance in the choice of treatments but also allows better prognostic counselling.

It has been generally accepted that the invasive and metastatic potential of HCC was mostly attributed to the individual clinicopathological and biological characteristics. The diversity of biological characteristics determines the different invasive and metastatic potential of HCC (Okuda, 1997). Based on this diversity, in our institute, HCC was distinct phenotypically to: solitary large hepatocellular carcinoma (SLHCC, diameter $>5 \mathrm{~cm}$, and one

*Correspondence: Dr L-Y Yang; E-mail: lianyueyang@hotmail.com Received 14 October 2003; revised 28 January 2004; accepted 5 February 2004; published online II May 2004 node), which often grows expansively with capsule or pseudocapsule formation and has a favourable biological behaviour, nodular hepatocellular carcinoma (NHCC, node number $\geqslant 2$ ) and small hepatocellular carcinoma ( $\mathrm{SHCC}$, diameter $\leqslant 5 \mathrm{~cm}$ ). Our previous study found that SLHCC, although it shared large size, had longterm survival after hepatic resection compared to NHCC, which implied different invasive and metastatic potential between SLHCC and NHCC. However, further investigation of different invasive and metastatic potential between them was anticipated.

RhoC-GTPase, a member of the Ras superfamily of small guanosine triphosphatases (GTPases), shuttles between inactive GDP- and active GTP-bound form and exhibits intrinsic GTPase activities. Activation of Rho protein leads to the assembly of the actin-myosin contractile filaments into focal adhesion complexes that lead to cell polarity and facilitate motility (Nobes and Hall, 1995; Kimura et al, 1996; Leung et al, 1996). Recently, emerging data suggest that RhoC gene may have a potential role in carcinogenesis and metastasis of tumour cells (Itoh et al, 1999; Clark et al, 2000). The expression level of RhoC in metastatic region of pancreatic cancer has been previously reported to be higher than primary lesion (Suwa et al, 1998). In addition, a genomic-wide analysis of gene expression revealed that RhoC gene was involved in vascular invasiveness of HCC (Okabe et al, 2001).

To gain a better understanding of the difference in invasion and metastasis between SLHCC and NHCC, we employed cDNA microarray technology to profile gene-expression patterns in these two subtypes of HCC. Among 668 genes that were differentially 
expressed between SLHCC and NHCC, RhoC gene was selected for further study because of its potential association with tumour metastasis.

\section{MATERIALS AND METHODS}

\section{Tissue specimens}

The study protocol was approved by the Ethics Committee of the Central South University. Fresh samples of HCC tissues and pericarcinomatous liver tissues (PCLT, $1 \mathrm{~cm}$ away from the carcinoma) were obtained from 25 (22 male and 3 female) patients with hepatocellular carcinoma who underwent hepatoectomy at Xiangya Hospital of Central South University (CSU). The specimens were immediately flash frozen in liquid nitrogen and stored at $-80^{\circ} \mathrm{C}$ for RT $-\mathrm{PCR}$ and Western blotting. The median age of the patients was 53 years, ranging from 28 to 73 years. All specimens obtained from hepatic resection were confirmed by pathological examination and clinicopathological parameters, such as tumour diameter, number of tumour node, tumour capsule, histopathological classification, vein invasion and extrahepatic metastatic lesion. On this basis, the total number of cases could be divided into three groups (SLHCC, $n=7$; NHCC, $n=15$; SHCC, $n=3$ ).

\section{RNA isolation}

Total RNA was extracted from frozen tissue specimens (30$100 \mathrm{mg}$ ) using TRIZOL (GIBCO BRL, Gaithersburg, USA) reagent, according to the instructions provided by the manufacturer. The quality was checked in $1 \%$ agarose gels and the concentration was measured using an ultraviolet Spectrophotometer (Biochrom Ltd, Cambridge, England) (Suwa et al, 1998). The UV wavelength was adjusted at $260 \mathrm{~nm}$.

\section{cDNA microarray}

cDNA microarray was carried out on 22 cases (7 SLHCC and 15 NHCC) of HCC specimens. Examination of quality and quantity for individual RNA was performed, and all RNA samples were eligible. As we investigated the differentially expressed genes between the SLHCC group and NHCC group, the individual RNA specimens in the same group were mixed equally to obtain $30 \mu \mathrm{g}$ total RNA, which was reversely transcribed using reverse transcriptase (GIBCO BRL, Gaithersburg, USA). The mixed RNA samples from SLHCC were labelled with $\mathrm{Cy}-5$ and those from NHCC were labelled with Cy-3. After incubation at $42^{\circ} \mathrm{C}$ for $2 \mathrm{~h}$, the reactions of the two samples (one with $\mathrm{Cy}-3$ and other with $\mathrm{Cy}-5$ ) were mixed and purified by using a filtration spin column (Promega, Madison, USA). The whole volume of the purified probe was denatured at $95^{\circ} \mathrm{C}$ for $2 \mathrm{~min}$ and was applied to the cDNA microarray slide containing 8464 genes, which was provided by United Gene Holdings (Biostar Genechip Inc., Shanghai, China). The control spots of non-human origin in cDNA microarray slide include the U2 RNA gene, Hepatitis C Virus coat protein gene and spotting solution alone without DNA. After hybridisation at $42^{\circ} \mathrm{C}$ for $18 \mathrm{~h}$, the slide was washed for $10 \mathrm{~min}$ each in solutions of $2 \times$ SCC and $0.2 \%$ SDS, $0.1 \% \times$ SCC and $0.2 \%$ SDS and $0.1 \% \times$ SCC. Finally, the cDNA microarray slide was scanned with a Scan Array 4000 Standard Biochip Scanning System (Packard Biochip Technologies, VA, USA) at two wavelengths to detect the emission of both $\mathrm{Cy} 5$ and $\mathrm{Cy} 3$. The acquired image was analysed using ImaGene 3.0 software (Bio Discovery, Los Angeles, USA). The intensities of each spot at the two wavelengths represent the quantity of $\mathrm{Cy} 5$ and $\mathrm{Cy} 3$, respectively, hybridised to each spot. Ratios of Cy5 to Cy3 were computed for each location. The overall intensities were normalised with a correction coefficient obtained using the ratio of 64 housekeeping genes. Genes were identified as differentially expressed if the absolute value of the natural logarithm of the ratios was $>0.69$. To minimise artefacts arising from low expression values, only genes with raw intensity values for both $\mathrm{Cy} 5$ and $\mathrm{Cy} 3$ of $>800$ counts were selected for differential analysis (Li et al, 2002).

\section{Reverse transcription and polymerase chain reaction (RT - PCR)}

Total RNA $(2 \mu \mathrm{g})$ was reversely transcribed in a final $25 \mu \mathrm{l}$ reaction volume at $37^{\circ} \mathrm{C}$ for $1 \mathrm{~h}$ by using $200 \mathrm{U}$ M-MULV reverse transcriptase (Promega, Madison, USA). PCR amplification was performed in a final volume of $50 \mu \mathrm{l}$ containing $5 \mu \mathrm{l}$ first-strand cDNA solution, $2 \mathrm{U}$ Taq polymerase (Sangon, Shanghai, China), $5 \mu \mathrm{l} 10 \times$ PCR reaction buffer, $10 \mu \mathrm{M}$ of dNTP (Sangon, Shanghai, China) and $10 \mathrm{pmol}$ of each $3^{\prime}$ and $5^{\prime}$ sequence-specific oligonucleotide primer (Sangon, Shanghai, China) for RhoC and $\beta_{2^{-}}$ microglobulin gene (positive control). The primer sequences were as follows: human RhoC, 5'-TCCTCATCGTCTTCAGCAAG- $3^{\prime}$ (forward), 5'-CTGCAATCCGAAAGAAGCTG-3' (reverse); and human $\beta_{2}$-microglobulin, $5^{\prime}$-ACCCCCACTGAAAAAGATGA-3' (forward), 5'-ATCTTCAAACCTCCATGATG-3' (reverse). The amplification was performed on a DNA Thermal cycler (PerkinElmer, Shelton, USA) with an initial denaturation at $94^{\circ} \mathrm{C}$ for $3 \mathrm{~min}$, followed by 32 cycles of denaturation at $94^{\circ} \mathrm{C}$ for $40 \mathrm{~s}$, annealing at $56^{\circ} \mathrm{C}$ for $30 \mathrm{~s}$ and extension at $72^{\circ} \mathrm{C}$ for $1 \mathrm{~min}$, and a final extension at $72^{\circ} \mathrm{C}$ for $10 \mathrm{~min}$. PCR products were electrophoresed on $1.7 \%$ agarose gels; the bands representing amplified products were visualised using ethidium bromide during the exposure to a UV transilluminator. The density of the bands on the gel was quantified by densitometric analysis. RhoC gene expression was presented by the relative intensity of the PCR product bands from target sequences to that from the $\beta_{2}$-microglobulin gene (Suwa et al, 1998).

\section{SDS-PAGE and Western blotting}

Tissues from HCC and PCLT were lysed in a lysis buffer; $20 \mathrm{~mm}$ Tris- $\mathrm{HCl} \mathrm{pH}=7.4,10 \mathrm{~mm} \mathrm{NaCl}, 1 \mathrm{~mm}$ EDTA pH $=8.0,1 \mathrm{~mm} \mathrm{MgCl}_{2}$, $1 \%$ NP-40, 0.1\% SDS, 0.01\% PMSF (Sigma, St Louis, MO, USA) and protease-inhibitor (Promega, Madison, USA). The lysates were centrifuged at $13000 \mathrm{~g}$ for $20 \mathrm{~min}$ at $4^{\circ} \mathrm{C}$, and the supernatants were stored at $-80^{\circ} \mathrm{C}$. Extracts equivalent to $100 \mu \mathrm{g}$ of total protein were separated by stacking gel (3.5\%) and SDS-PAGE (12.5\%) separating gel with Tris-glycine system at $200 \mathrm{~V}$ for $1 \mathrm{~h}$. Blotting was performed to polyvinylidene fluoride membrane (Sigma, St Louis, MO, USA) at $100 \mathrm{~V}$ for $1 \mathrm{~h}$ in a tank of transfer buffer (48 mm Tris- $\mathrm{HCl}, 192 \mathrm{~mm}$ glycine, $20 \%$ methanol $\mathrm{pH}=8.4$ ). The membranes were blocked in $4 \%$ nonfat dry milk in PBS containing $0.1 \%$ Tween- 20 for $1 \mathrm{~h}$ at room temperature. Then, the membranes were incubated with primary antibody (goat anti-human RhoC polyclonal antibody, Santa Cruz, CA, USA, diluted at $1: 500$ ) for $1 \mathrm{~h}$ at $37^{\circ} \mathrm{C}$. After washing, the membranes were incubated with a $1: 1000$ dilution of horseradish peroxidase-linked rabbit anti-goat antibody (Santa Cruz, CA, USA) for $30 \mathrm{~min}$ at $37^{\circ} \mathrm{C}$. Then the membranes were washed and treated with Western blotting luminal reagent (Santa Cruz, CA, USA) to visualise the bands; the results were obtained on Kodak film and quantified by densitometry (Beckman, South Pasadena, Canada) (Abraham et al, 2001).

\section{Immunohistochemistry}

A total of 94 HCC specimens, including the 25 cases of HCC fresh specimens (used for RT-PCR and Western blotting), were evaluated for immunohistochemistry. All the specimens were collected from Xiangya Hospital of Central South University between 1994 and 2002. Follow-up data were obtained following operation for all patients; the end point of follow-up was set at the 
patient's death. In brief, tissue sections of $4 \mu \mathrm{m}$ thick were cut and baked at $60^{\circ} \mathrm{C}$ for $2 \mathrm{~h}$, deparaffinised in xylene and rehydrated through graded ethanol. Next, $3 \%$ hydrogen peroxide was applied to block the endogenous peroxidases for $20 \mathrm{~min}$ and sections were subjected to heat-induced antigen retrieval in $0.01 \mathrm{M}$ citrate buffer $(\mathrm{pH}=6.0)$. The sections were incubated with normal goat serum to reduce nonspecific binding. Then, they were incubated at $37^{\circ} \mathrm{C}$ for $1 \mathrm{~h}$ with specific antibodies (goat anti-human RhoC polyclonal antibody, Santa Cruz, CA, USA) used at a $1: 100$ dilution. The second antibody was applied for $45 \mathrm{~min}$ at $37^{\circ} \mathrm{C}$. The streptavidin biotin-peroxidase complex (SABC) tertiary system (Boster, Wuhan, China) was used according to the manufacturer's instruction for $20 \mathrm{~min}$ at room temperature. The tissues were visualised by applying 3,3-diaminobenzidine tetrahydrochloride (DAB) for 3 min. Sections were counterstained using haematoxylin, dehydrated through gradient alcohols and mounted for viewing. Negative controls were carried out by omitting the primary antibody, whereas RhoC overexpression confirmed by Western blotting was used as positive controls. The intensity of cytoplasmic staining was scored as 0 to $3+$ by comparison to the positive controls. The scoring system has been previously validated (Kleer et al, 2002). Diffuse, moderate to strong cytoplasmic staining characterised RhoC-positive expression (scores $2+$ and $3+$ ). RhoC-negative expressions were devoid of any cytoplasmic staining or contained faint, equivocal staining (scores 0 and $1+$, Figure 1)
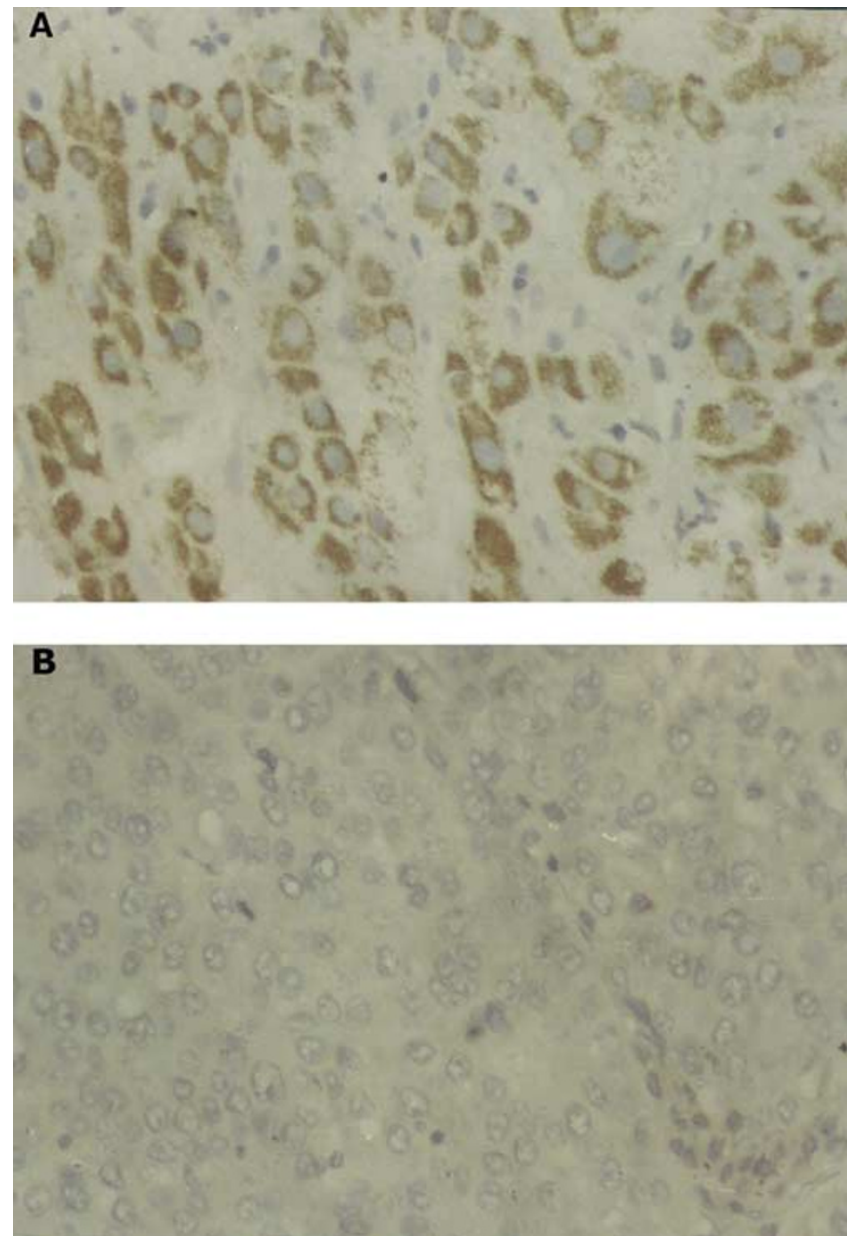

Figure I (A) Typical staining for positive cytoplastic RhoC expression in an HCC (original magnification $\times 400$ ). (B) Typical staining for negative cytoplastic RhoC expression in an HCC (original magnification $\times 400$ )

\section{Statistical analysis}

Statistical analysis was performed using the SPSS (version 11.0, Chicago, IL, USA). The Fisher's exact test was performed to access differences in clinicopathological parameters between SLHCC and NHCC. Spearman's correlation coefficient was used to examine the relationship between expression levels of RhoC mRNA and protein. The nonparametric Mann-Whitney test was monitored to evaluate the differences in the expression of RhoC between groups, and also to analyse the correlation between the expression of RhoC and clinicopathological variables. Postoperative survival was analysed by the log-rank test. Differences were considered significant when $P<0.05$.

\section{RESULTS}

\section{Clinical and pathological characterisation of SLHCC and NHCC}

According to the criteria described in the introduction, there were seven cases of SLHCC and 15 cases of NHCC among the total 25 cases of HCC studied. Most of the SLHCC cases were differentiated at I-II (five out of seven, 71\%), whereas considerably fewer cases of NHCC (2 out of 15,13\%) exhibited the same level of differentiation. In addition, there was a substantial difference in tumour vein invasion with a significantly higher percent in NHCC than in SLHCC (one out of seven, 14\% SLHCC vs 11 out of $15,73 \%$ NHCC). There were five cases of SLHCC, but only three cases of NHCC, which exhibited capsulation (5 out of 7, 71\% SLHCC $v s 12$ out of $15,20 \%$ NHCC) also. The distribution of sex, liver cirrhosis in each group showed no significant differences (Table 1).

\section{Evaluation of invasiveness score for HCC}

Invasiveness score (IS) was applied to evaluate the invasive and metastatic abilities of SLHCC and NHCC (El-Assal et al, 1997). Three cases of SLHCC (three out of seven) and three cases of NHCC (three out of 15) scored IS $\leqslant 1$ (low invasive); three cases of SLHCC (three out of seven) and five cases NHCC (five out of 15) scored $2 \leqslant I S \leqslant 4$ (moderate invasive); and one case of SLHCC (one out of seven) and seven cases of NHCC (seven out of 15) scored IS $\geqslant 5$ (high invasive). Collectively, the SLHCC group showed lower IS scores than the NHCC group $(P=0.02)$, suggesting that SLHCC is less invasive than NHCC.

Table I Clinicopathological differences between SLHCC and NHCC

\begin{tabular}{lccc}
\hline Variables & SLHCC (n) & NHCC (n) & Fisher's test \\
\hline Sex & 6 & 14 & $P=1.00$ \\
Male & 1 & 1 & \\
Female & & & \\
Liver cirrhosis & 5 & 6 & \\
$\quad$ Present & 2 & 9 & \\
Absent & & & \\
Cell differentiation & 5 & 2 & \\
I-II & 2 & 13 & \\
III-IV & 2 & & \\
Capsule formation & 5 & 3 & \\
Present & & 12 & \\
Absent & 2 & & \\
Vein invasion & & 11 & \\
Present & 1 & 4 & \\
$\quad$ Absent & 6 & & \\
\hline
\end{tabular}




\section{Identification of genes differentially expressed in SLHCC} and NHCC

The distinct phenotypes displayed by SLHCC and NHCC implicate differences in biology. To gain a better understanding of the differences between SLHCC and NHCC, we made an attempt to profile gene expression patterns in these two types of HCC using cDNA microarray. By setting the threshold value of 2 (upregulation) and 0.5 (downregulation) for assessing the difference in the expression level between SLHCC and NHCC, 668 out of 8464 human genes (cDNA microarray, Biostar Genechip Inc., Shanghai, China) were differentially expressed (355 upregulated and 313 downregulated genes). The altered genes clustered into different subsets of cellular genes whose products are implicated in biological activities generally consistent with the physiology and pathology of SLHCC and NHCC. A partial list of the genes differentially expressed is shown in Table 2 . Complete information of the cDNA microarray can be accessed through our website. Collectively, the array data provide a genome-wide view of differences in gene expression between SLHCC and NHCC. Of particular interest among 668 genes that were differentially expressed is the RhoC gene, whose expression is higher in highly invasive NHCC than in less invasive SLHCC since RhoC expression has been implicated in tumour metastasis. We therefore decided to validate the array data by assessing the expression of RhoC at the level of message RNA and protein in the HCC samples.

\section{Expression of RhoC mRNA and protein}

The expressions of RhoC mRNA and protein were detected in all HCC tissues and PCLT. However, the levels of both RhoC mRNA and protein are significantly higher in HCC tissues than in PCLT $(P=0.001)$. Significantly, we detected substantially higher levels of both RhoC mRNA and protein in extrahepatic HCC tissues than those in intrahepatic HCC tissues $(P=0.009$ and 0.002 , Figures 2 and 3$)$. Statistical analyses revealed a strongly positive correlation $(r=0.735, P<0.001$, Figure 4$)$.

\section{Correlation between RhoC expression levels and clinicopathological parameters of HCC}

The distribution pattern of RhoC was further analysed by dividing the expression levels of RhoC mRNA and protein in subgroups according to the clinicopathological parameters. Interestingly, the RhoC mRNA and protein expression levels in HCC with multinodes were significantly higher than those with one node $(P=0.011$ and 0.015$)$. Furthermore, increased RhoC expression strongly correlates with vein invasion $(P=0.006$ and 0.030$)$. A significant difference in RhoC expression was also evident between HCC with poor differentiation and those with well differentiation $(P=0.004$ and 0.002$)$. There were no significant associations between expression of RhoC gene and other clinicopathological parameters such as sex, HBV infection, liver cirrhosis, tumour size and capsular condition (Table 3 ).

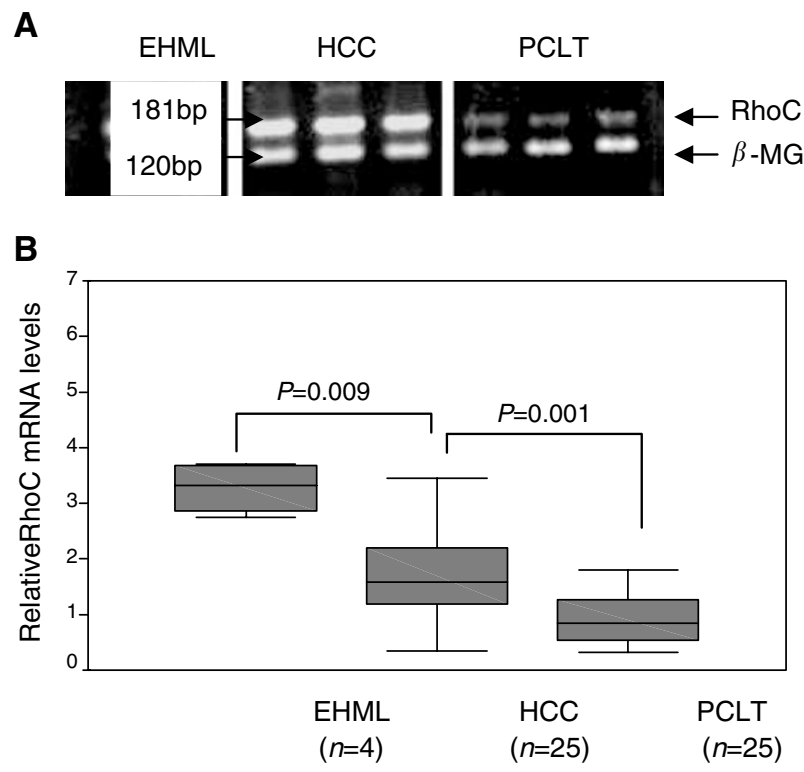

Figure 2 Detection of RhoC mRNA by RT-PCR. (A) PCR products were visualised by ethidium bromide staining. Eight selected samples from each group are shown: RhoC (I8I bp); $\beta$-MG (I20 bp); EHML: extrahepatic metastatic lesions; HCC: hepatocellular carcinoma tissues; PCLT: pericarcinomatous liver tissues. (B) The Mann-Whitney test was performed to compare the mRNA expression of RhoC between different groups; EHML showed a significantly higher mRNA expression level than HCC and the same between HCC and PCLT.

Table 2 Parts of genes differentially expressed between SLHCC and NHCC

\begin{tabular}{|c|c|c|c|}
\hline GeneBank ID & Gene name & Function & Ratio (Cy5/Cy3) \\
\hline NM_031934 & Homo sapiens RAB34, member RAS oncogene family (RAB34), mRNA & $\begin{array}{l}\text { Cellular skeleton and } \\
\text { movement }\end{array}$ & 0.224 \\
\hline NM_003177 & Homo sapiens spleen tyrosine kinase (SYK), mRNA & Signal transduction & 0.240 \\
\hline NM_014624 & Homo sapiens SI00 calcium-binding protein A6 (calcyclin) (SI00A6), mRNA & Signal transduction & 0.345 \\
\hline NM_022807 & $\begin{array}{l}\text { Homo sapiens small nuclear ribonucleoprotein polypeptide N (SNRPN), transcript variant 4, } \\
\text { mRNA }\end{array}$ & Growth and development & 0.389 \\
\hline NM_001 168 & Homo sapiens baculoviral IAP repeat-containing 5 (survivin) (BIRC5), mRNA & Cellular apoptosis & 0.441 \\
\hline NM_I75744 & Homo sapiens ras homologue gene family, member $\mathrm{C}$ (ARHC), mRNA & $\begin{array}{l}\text { Cellular skeleton and } \\
\text { movement }\end{array}$ & 0.463 \\
\hline NM_002530 & Homo sapiens neurotrophic tyrosine kinase, receptor, type 3 (NTRK3), mRNA & Growth and development & 0.464 \\
\hline NM_001912 & Homo sapiens cathepsin L (CTSL), mRNA & Matrix degrade & 0.477 \\
\hline NM_000210 & Homo sapiens integrin, alpha 6 (ITGA6), mRNA & Matrix degrade & 0.493 \\
\hline NM_004394 & Homo sapiens death-associated protein (DAP), mRNA & Cellular apoptosis & 2.014 \\
\hline NM_001901 & Homo sapiens connective tissue growth factor (CTGF), mRNA & Growth and development & 2.026 \\
\hline NM_003445 & Homo sapiens zinc finger protein I55 (pHZ-96) (ZNFI55), mRNA & Signal transduction & 2.095 \\
\hline NM_000389 & Homo sapiens cyclin-dependent kinase inhibitor IA (p2I, Cip I) (CDKNIA), mRNA & Cellular apoptosis & 2.292 \\
\hline NM_017675 & Homo sapiens protocadherin LKC (PC-LKC), mRNA & Matrix degrade & 2.358 \\
\hline AF22̄0656 & Homo sapiens apoptosis-associated nuclear protein PHLDAI (PHLDAI) mRNA, partial cds & Cellular apoptosis & 2.388 \\
\hline NM_00I880 & Homo sapiens activating transcription factor 2 (ATF2), mRNA & Cellular apoptosis & 3.090 \\
\hline NM_001257 & Homo sapiens cadherin I3, H-cadherin (heart) (CDHI3), mRNA & Matrix degrade & 4.60 \\
\hline
\end{tabular}


A

EHML

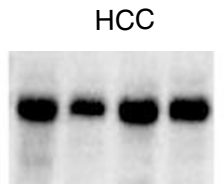

B

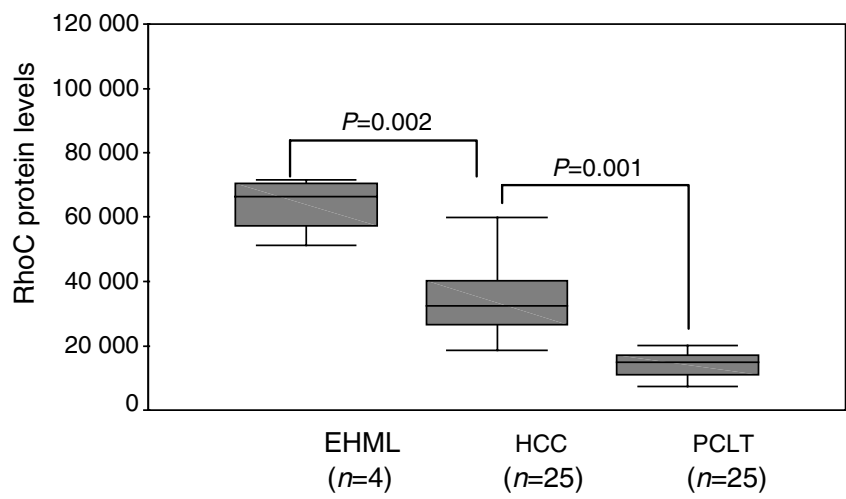

Figure 3 Western analysis of RhoC protein. (A) The protein expression levels were obtained on Kodak films and were quantified by densitometry. A total of 10 selected samples were shown. (B) The Mann-Whitney test showed significant differences between EHML and HCC, and also between HCC and PCLT.

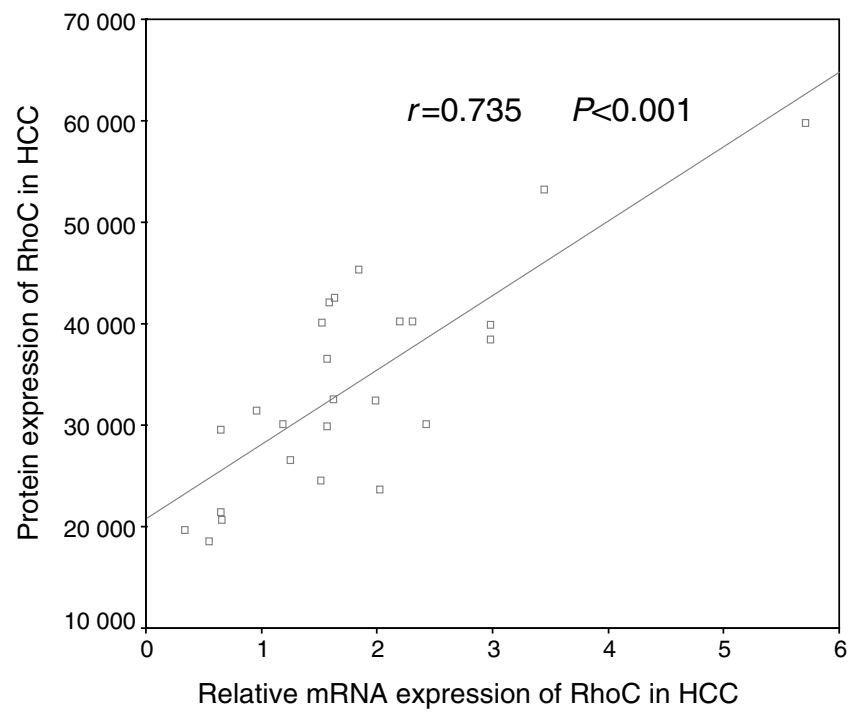

Figure 4 Correlation between mRNA and protein expression levels of RhoC in HCC was evaluated by Spearman's correlation coefficient. RhoC Protein levels were directly correlated with the levels of RhoC mRNA in $\mathrm{HCC}$ with adjusted $r_{\mathrm{s}}=0.735$, and two-tailed probability, $\mathrm{P}<0.00 \mathrm{I}$.

\section{Prognostic implications of RhoC expression}

Kaplan-Meier survival curves for patients with HCC, categorised according to RhoC expression, are shown in Figure 5. The mean survival of patients with RhoC-positive expression, which was determined by immunohistochemical staining (Figure 5), was significantly lower (356 days) than that of patients with RhoCnegative expression ( 579 days, $P=0.0031$ ), indicative of a positive correlation of RhoC expression with poor prognosis.
Table 3 Relationship between expression level of RhoC and clinicopathological parameters

P-value

\begin{tabular}{|c|c|c|c|}
\hline Variables & No. of patients & RhoC mRNA & RhoC protein \\
\hline \multicolumn{4}{|l|}{ Sex } \\
\hline Male & 22 & 0.675 & 0.452 \\
\hline Female & 3 & & \\
\hline \multicolumn{4}{|l|}{ Liver cirrhosis } \\
\hline Present & 14 & 0.584 & 0.622 \\
\hline Absent & 11 & & \\
\hline \multicolumn{4}{|c|}{ Capsule formation } \\
\hline Present & 13 & 0.785 & 0.744 \\
\hline Absent & 12 & & \\
\hline \multicolumn{4}{|c|}{ Tumour node } \\
\hline$\geqslant 2$ & 15 & 0.011 & 0.015 \\
\hline$<2$ & 10 & & \\
\hline \multicolumn{4}{|c|}{ Cell differentiation } \\
\hline $1-11$ & 9 & 0.004 & 0.002 \\
\hline III-IV & 16 & & \\
\hline \multicolumn{4}{|l|}{ Vein invasion } \\
\hline Present & 13 & 0.006 & 0.030 \\
\hline Absent & 12 & & \\
\hline \multicolumn{4}{|c|}{ Extrahepatic metastasis } \\
\hline Present & 5 & 0.103 & 0.077 \\
\hline Absent & 20 & & \\
\hline \multicolumn{4}{|c|}{ Tumour size (cm) } \\
\hline$>5$ & 18 & 0.287 & 0.904 \\
\hline$\leqslant 5$ & 7 & & \\
\hline
\end{tabular}

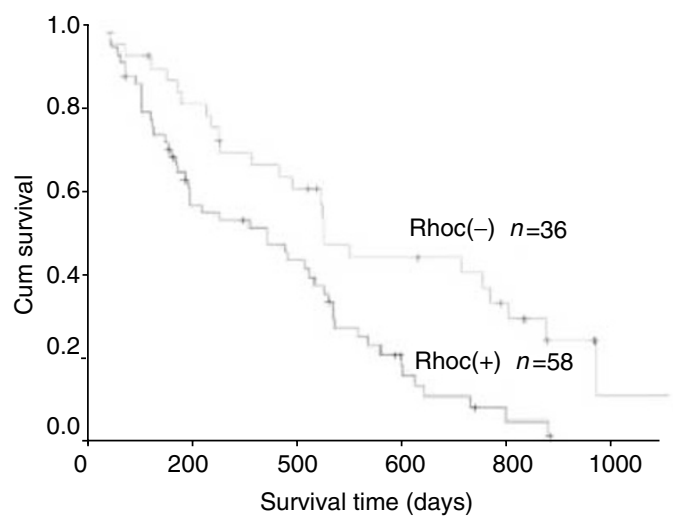

Figure 5 Kaplan-Meier survival curves for RhoC-positive expression group $(n=58)$ and RhoC-negative expression group $(n=36)$ based on the results of immunohistochemistry. HCC patients with RhoC-positive expression revealed a significantly poor prognosis than those with RhoCnegative expression (log-rank test, $P=0.003 \mathrm{I}$ ).

\section{DISCUSSION}

Classification and prognosis of HCC has been traditionally based on the size of HCC; the large HCC is often considered as advance and unresectable. However, we have observed that there is one type of HCC, which is large in size but exhibits a lower invasive and metastatic potential. This type of HCC typically has just single node and we defined them SLHCC. On the contrary, 
there is another type of HCC in which invasion and metastasis often occur early despite their small size. This type of HCC, however, has usually more than one node. These observations of ours are consistent with the sixth TNM classification for HCC revised by UICC that the number of nodes, but not size of HCC, is the crucial factor for classification and prognosis of HCC (Poon and Fan, 2003). We routinely divide HCC, based on the distinct phenotype, into: SLHCC (diameter $>5 \mathrm{~cm}$, only one node), NHCC (node number $\geqslant 2$ ) and SHCC (diameter $\leqslant 5 \mathrm{~cm}$ ). Our clinical data indicate that NHCC displays a significantly greater potency in invasion and metastasis than SLHCC does. In keeping with the notion that cell differentiation status and vein invasion highly correlate with the invasion and metastasis of HCC (Liver Cancer Study Group of Japan, 1990; Farinati et al, 2000; Ohkubo et al, 2000; Utsunomiya et al, 2000), we found that SLHCC is generally better differentiated and has less vein invasion than NHCC. SLHCC is also prone to formulate capsulation. Together with the IS, our data indicate that NHCC in general has a greater propensity to undergo metastasis than SLHCC does.

The distinct phenotypes exhibited by SLHCC and NHCC would likely be attributable to differences in gene expression. Our microarray analyses indicate that it is indeed the case. Among 8464 human genes examined, a total of 668 genes are differentially expressed between SLHCC and NHCC. While further studies are required to examine how those genes might contribute to the pathology of HCC, the difference in gene expression may represent the molecular basis underlying the distinct phenotypes exhibited by NHCC and SLHCC.

Owing to its role in cytoskeletal reorganisation, in focal adhesion contacts (Nobes and Hall, 1995; Kimura et al, 1996; Leung et al, 1996) and in tumour invasion (Clark et al, 2000), RhoC was chosen from the pool of 668 differentially expressed genes for further analysis of the possibility that increased RhoC expression might correlate with HCC metastasis. Three different approaches, RT-PCR to measure mRNA levels, Western and immunohistochemical staining to determine protein levels, were employed to verify the expression of RhoC. Our data indicate that the expression of RhoC was significantly higher in HCC tissues than that in the corresponding PCLT. Furthermore, increased expression of RhoC in HCC seemed to correlate positively with poor cell differentiation and with tumour vein invasion. It is well documented that HCC often undergo dedifferentiation (from well-differentiated to poorly differentiated) during multistep tumour progression (Mise et al, 1998), and that some HCC acquire metastatic potential during this progression, resulting in vein invasion (Hui et al, 1999). Our results suggest that increased expression of RhoC is closely associated with the later events in liver carcinogenesis.
Our present study also showed that extrahepatic metastasis of HCC expressed significantly higher RhoC levels than intrahepatic HCC, and elevated expression of RhoC positively correlated with vein invasion, and the number of tumour nodes. Similar observations have been reported in other types of tumours. For instance, overexpression of RhoC was found in metastatic lesions of inflammatory breast cancer and pancreatic ductal adenocarcinoma (Suwa et al, 1998; Fritz et al, 2002; Kleer et al, 2002), and upregulation was associated with tumour progression in ovarian carcinoma (Horiuchi et al, 2003). While the molecular mechanism by which RhoC facilitates tumour metastasis remains to be determined, it is possible that increased expression of RhoC could result in: (1) disruption of cell polarity, which plays an important role in the epithelial-mesenchymal transition observed in more aggressive tumours (Zondag et al, 2000); (2) contribution to the loss of adherens junctions (Braga et al, 2000); (3) increase motility and ability to remould the extra-cellular matrix, which is required for tumour cells to become locally invasive (Khanna et al, 2001); and (4) increase in angiogenic factors that would result in promotion of vascularisation in tumour and increase the likelihood of tumour cell entering the bloodstream (van Golen et al, 2000).

Our data suggest a possibility that RhoC expression could be used as a potential prognostic marker for HCC patients. To test this possibility, anti-RhoC immunohistochemical staining was performed on 94 cases of HCC that included the 25 cases mentioned above. Owing to the lower detection sensitivity of the immunohistochemistry method, some of the 94 cases of HCC showed negative staining of RhoC, despite the fact that RhoC protein can be detected in all 25 cases of HCC with Western blotting. Nevertheless, when we divided the total cases of HCC into either a RhoC-positive or -negative group, the RhoC-negative HCC patients in general had a better prognosis than the RhoC-positive HCC patients. Together, our data strongly suggest that increased RhoC expression in HCC correlates with a poor prognosis.

\section{ACKNOWLEDGEMENTS}

We thank Zhi-min Yuan (Department of Cancer Cell Biology, Harvard School of Public Health, Boston, MA, USA), Min-Juan Lin (Department of Respiratory Medicine, Xiangya Hospital, Central South University) and Dr AM Abd EI-Aty (Pharmacogenetics Research Institute, Institute of Clinical Pharmacology, Central South University) for their critical and useful comments on the manuscript. This work was supported by the National Key Technologies $\mathrm{R}$ and D Program Grant No. 2001BA703B04 and Hunan Province Developing Planning Committee Grant No. 2001-907.

\section{REFERENCES}

Abraham MT, Kuriakose MA, Sacks PG, Yee H, Chiriboga L, Bearer EL, Delacure MD (2001) Motility-related proteins as markers for head and neck squamous cell cancer. Laryngoscope 111: 1285-1289

Akriviadis EA, Llovet JM, Efremidis SC, Shouval D, Canelo R, Ringe B, Meyers WC (1998) Hepatocellular carcinoma. Br J Surg 85: 1319-1331 Braga VM, Betson M, Li X, Lamarche VN (2000) Activation of the small GTPase Rac is sufficient to disrupt cadherin-dependent cell-cell adhesion in normal human keratinocytes. Mol Biol Cell 11: 3703-3721

Clark EA, Golub TR, Lander ES, Hynes RO (2000) Genomic analysis of metastasis reveals an essential role for RhoC. Nature 406: 532-535

El-Assal ON, Yamanoi A, Soda Y, Yamaguchi M, Yu L, Nagasue N (1997) Proposal of invasiveness score to predict recurrence and survival after curative hepatic resection for hepatocellular carcinoma. Surgery 122: $571-577$
Fan ST, Lo CM, Liu CL, Lam CM, Yuen WK, Yeung C, Wong J (1999) Hepatectomy for hepatocellular carcinoma: toward zero hospital deaths. Ann Surg 229: $322-330$

Farinati F, Rinaldi M, Gianni S, Naccarato R 2000 How should patients with hepatocellular carcinoma be staged? Validation of a new prognostic system. Cancer 89: 2266-2273

Fritz G, Brachetti C, Bahlmann F, Schmidt M, Kaina B (2002) Rho GTPases in human breast tumours: expression and mutation analyses and correlation with clinical parameters. $\mathrm{Br} J$ Cancer 87: $635-644$

Horiuchi A, Imai T, Wang C, Ohira S, Feng Y, Nikaido T, Konishi I (2003) Up-regulation of small GTPases, RhoA and RhoC, is associated with tumor progression in ovarian carcinoma. Lab Invest 83: $861-870$ 
Hui AM, Li X, Makuuchi M, Takayama T, Kubota K (1999) Over-expression and lack of retinoblastoma protein are associated with tumor progression and metastasis in hepatocellular carcinoma. Int J Cancer 84: $604-608$

Itoh K, Yoshioka K, Akedo H, Uehata M, Ishizaki T, Narumiya S (1999) An essential part for Rho-associated kinase in the transcellular invasion of tumor cells. Nat Med 5: 221-225

Khanna C, Khan J, Nguyen P, Prehn J, Caylor J, Yeung C, Trepel J, Meltzer P, Helman L (2001) Metastasis-associated differences in gene expression in a murine model of osteosarcoma. Cancer Res 61: 3750-3759

Kimura K, Ito M, Amano M, Chihara K, Fukata Y, Nakafuku M, Yamamori B, Feng J, Nakano T, Okawa K, Iwamatsu A, Kaibuchi K (1996) Regulation of myosin phosphatase by Rho and Rho-associated kinase (Rho-kinase). Science 273: $245-248$

Kleer CG, van Golen KL, Zhang Y, Wu ZF, Rubin MA, Merajver SD (2002) Characterization of RhoC expression in benign and malignant breast disease: a potential new marker for small breast carcinomas with metastatic ability. Am J Pathol 160: 579-584

Leung T, Chen XQ, Manser E, Lim L (1996) The p160 RhoA-binding kinase ROK alpha is a member of a kinase family and is involved in the reorganization of the cytoskeleton. Mol Cell Biol 16: $5313-5327$

Li Y, Li Y, Tang R, Xu H, Qiu M, Chen Q, Chen J, Fu Z, Ying K, Xie Y, Mao Y 2002 Discovery and analysis of hepatocellular carcinoma genes using cDNA microarrays. J Cancer Res Clin Oncol 128: 369-379

Liver Cancer Study Group of Japan (1990) Primary liver cancer in Japan. Clinicopathologic features and results of surgical treatment. Ann Surg 211: $277-287$

Makuuchi M, Takayama T, Kubota K, Kimura W, Midorikawa Y, Miyagawa S, Kawasaki S (1998) Hepatic resection for hepatocellular carcinoma Japanese experience. Hepatogastroenterology 45: 1267-1274

Mise K, Tashiro S, Yogita S, Wada D, Harada M, Fukuda Y, Miyake H, Isikawa M, Izumi K, Sano N (1998) Assessment of the biological malignancy of hepatocellular carcinoma: relationship to clinicopathological factors and prognosis. Clin Cancer Res 4: 1475-1482

Murray CJ, Lopez AD (1997) Mortality by cause for eight regions of the world: Global Burden of Disease Study. Lancet 349: 1269-1276
Nobes CD, Hall A (1995) Rho, rac, and cdc42 GTPases regulate the assembly of multimolecular focal complexes associated with actin stress fibers, lamellipodia, and filopodia. Cell 81: 53-62

Ohkubo T, Yamamoto J, Sugawara Y, Shimada K, Yamasaki S, Makuuchi M, Kosuge T (2000) Surgical results for hepatocellular carcinoma with macroscopic portal vein tumor thrombosis. J Am Coll Surg 191: 657-660

Okabe H, Satoh S, Kato T, Kitahara O, Yanagawa R, Yamaoka Y, Tsunoda T, Furukawa Y, Nakamura Y (2001) Genome-wide analysis of gene expression in human hepatocellular carcinomas using cDNA microarray: identification of genes involved in viral carcinogenesis and tumor progression. Cancer Res 61: 2129-2137

Okuda K (1997) Hepatocellular carcinoma: clinicopathological aspects. J Gastroenterol Hepatol 12: S314-318

Poon RT, Fan ST (2003) Evaluation of the new AJCC/UICC staging system for hepatocellular carcinoma after hepatic resection in Chinese patients. Surg Oncol Clin N Am 12: 35 - 50

Poon TPR, Fan ST, Wong J (2000) Risk factors, prevention, and management of postoperative recurrence after resection of hepatocellular carcinoma. Ann Surg 232: 10-24

Schafer DF, Sorrell MF (1999) Hepatocellular carcinoma. Lancet 353: 1253 1256

Suwa H, Ohshio G, Imamura T, Watanabe G, Arii S, Imamura M, Narumiya S, Hiai H, Fukumoto M (1998) Overexpression of the rhoC gene correlates with progression of ductal adenocarcinoma of the pancreas. $\mathrm{Br}$ J Cancer 77: $147-152$

Utsunomiya T, Shimada M, Taguchi KI, Hasegawa H, Yamashita Y, Hamatsu T, Aishima SI, Sugimachi K (2000) Clinicopathologic features and postoperative prognosis of multicentric small hepatocellular carcinoma. J Am Coll Surg 190: $331-335$

van Golen KL, Wu ZF, Qiao XT, Bao L, Merajver SD (2000) RhoC GTPase overexpression modulates induction of angiogenic factors in breast cells. Neoplasia 2: $418-425$

Zondag GC, Evers EE, ten Klooster JP, Janssen L, van der Kammen RA, Collard JG (2000) Oncogenic Ras downregulates Rac activity, which leads to increased Rho activity and epithelial-mesenchymal transition. J Cell Biol 149: $775-782$ 